\title{
First results from a prototype of the Fluorescence detector Array of Single-pixel Telescopes
}

\author{
Toshihiro Fujii $^{*, a, b}$, Max Malacari ${ }^{c}$, Mario Bertaina ${ }^{d}$, Marco Casolino ${ }^{e}$, Bruce \\ Dawson $^{c}$, Pavel Horvath $^{f}$, Miroslav Hrabovsky $^{f}$, Jiaqi Jiang ${ }^{a}$, Dusan Mandat ${ }^{g}$, Ariel \\ Matalon $^{a}$, John N. Matthews ${ }^{h}$, Pavel Motloch $^{a}$, Miroslav Palatka $^{g}$, Miroslav Pech ${ }^{g}$, \\ Paolo Privitera $^{a}$, Petr Schovanek $^{g}$, Yoshiyuki Takizawa ${ }^{e}$, Stan B. Thomas ${ }^{h}$, Petr \\ Travnicek $^{g}$, Katsuya Yamazaki ${ }^{i}$ \\ ${ }^{a}$ Kavli Institute for Cosmological Physics, University of Chicago, Chicago, Illinois, USA \\ ${ }^{b}$ Institute for Cosmic Ray Research, University of Tokyo, Kashiwa, Chiba, Japan \\ ${ }^{c}$ Department of Physics, University of Adelaide, Adelaide, S.A., Australia \\ ${ }^{d}$ Dipartimento di Fisica, Università di Torino and INFN Torino, Torino, Italy \\ ${ }^{e}$ RIKEN Advanced Science Institute, Wako, Saitama, Japan \\ ${ }^{f}$ Palacky University, RCPTM, Olomouc, Czech Republic \\ ${ }^{g}$ Institute of Physics of the Academy of Sciences of the Czech Republic, Prague, Czech Republic \\ ${ }^{h}$ High Energy Astrophysics Institute and Department of Physics and Astronomy, University of \\ Utah, Salt Lake City, Utah, USA \\ ${ }^{i}$ Earthquake Research Institute, The University of Tokyo, Tokyo, Japan \\ E-mail: Eujiiekicp.uchicago.edu
}

\begin{abstract}
We present a concept for large-area, low-cost detection of ultra-high energy cosmic rays (UHECRs) with a Fluorescence detector Array of Single-pixel Telescopes (FAST), addressing the requirements for the next generation of UHECR experiments. In the FAST design, a large field of view is covered by a few pixels at the focal plane of a mirror or Fresnel lens. We report first results of a FAST prototype installed at the Telescope Array site, consisting of a single $200 \mathrm{~mm}$ photomultiplier tube at the focal plane of a $1 \mathrm{~m}^{2}$ Fresnel lens system taken from the prototype of the JEM-EUSO experiment. The FAST prototype took data for 19 nights, demonstrating remarkable operational stability. We detected laser shots at distances of several kilometres as well as 16 highly significant UHECR shower candidates.
\end{abstract}

The 34th International Cosmic Ray Conference,

30 July- 6 August, 2015

The Hague, The Netherlands

\footnotetext{
* Speaker.
} 


\section{Introduction}

The origin and nature of ultra-high energy cosmic rays (UHECRs) is one of the most intriguing mysteries in particle astrophysics [四]. Given their minute flux, less than one per century per square kilometre at the highest energies, a very large area must be instrumented to collect significant statistics. The energy, arrival direction, and mass composition of UHECRs can be inferred from studies of the cascade of secondary particles (Extensive Air Shower, EAS) produced by their interaction with the Earth's atmosphere. Two well-established techniques are used for UHECR detection: 1) arrays of detectors (e.g. plastic scintillators and water-Cherenkov stations) sample EAS particles reaching the ground; 2) large field of view telescopes allow for reconstruction of the shower development in the atmosphere by imaging UV fluorescence light from atmospheric nitrogen excited by EAS particles.

The Pierre Auger Observatory (Auger) [] $]$ and the Telescope Array Experiment (TA) [B] [四], the two largest UHECR experiments currently in operation, combine the two techniques, with arrays of particle detectors overlooked by fluorescence detector (FD) telescopes. Auger covers an area of over 3,000 $\mathrm{km}^{2}$ close to the town of Malarguie in the province of Mendoza, Argentina. TA is located near the town of Delta in central Utah, USA, and covers an area of $700 \mathrm{~km}^{2}$. Significant advances in our understanding of UHECRs have been achieved in the last decade by these experiments [ []]. However, these results are limited by statistics at the highest energies. To further advance the field, the next generation of experiments will require an aperture which is larger by an order of magnitude. This may be accomplished by fluorescence detection of UHECR showers from space, as in the proposed JEM-EUSO [6] mission, or with a ground array much larger than Auger. Low-cost, easily-deployable detectors will be essential for a ground-based experiment.

In this paper, we present an FD telescope concept which would fulfill these requirements. The Fluorescence detector Array of Single-pixel Telescopes (FAST) would consist of compact FD telescopes featuring a smaller light-collecting area and many fewer pixels than current FD designs, leading to a significant reduction in cost.

\section{FAST concept and prototype at the Telescope Array site}

In the FAST design, $\mathrm{a} \sim 30^{\circ} \times 30^{\circ}$ field of view (FOV) is covered by just a few $200 \mathrm{~mm}$ PMTs at the focal plane of a mirror or Fresnel lens of $A \sim 1 \mathrm{~m}^{2}[\mathbb{[}]$. We expect a significant cost reduction thanks to FAST's compact design with smaller light-collecting optics, a smaller telescope housing, and a small number of PMTs and associated electronics. FAST stations, powered by solar panels and with wireless connection, could be deployed in an array configuration to cover a very large area at low cost.

A first test of the FAST concept was performed profiting from the existing infrastructure of the JEM-EUSO experiment at the TA site in Utah, USA, where a prototype [ [ $]$ ] is currently installed for a comprehensive test of the optics and electronics of this space-based detector. The light-collecting area $\left(\sim 1 \mathrm{~m}^{2}\right)$ and circular FOV $\left(\sim 7^{\circ}\right.$ radius) of the JEM-EUSO prototype telescope (EUSO-TA telescope) are close to the FAST reference design (for a single pixel), providing a perfect test bed for the FAST concept. 


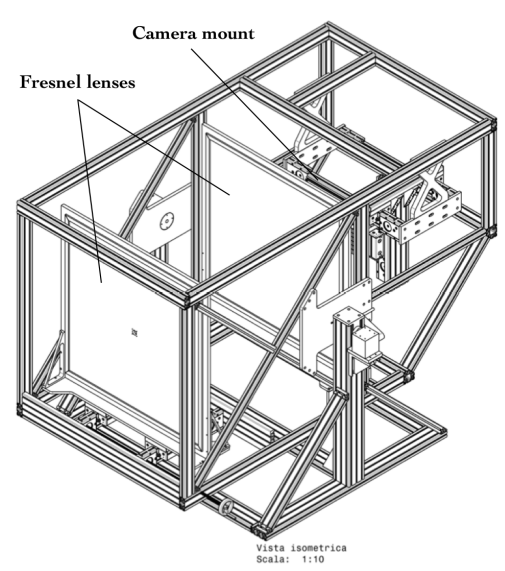

(a)

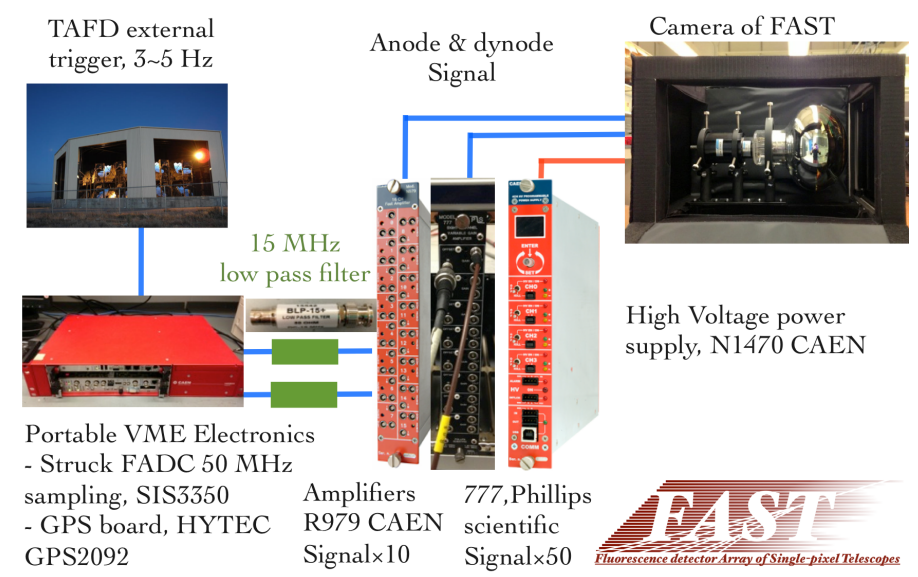

(b)

Figure 1: The EUSO-TA telescope optics (a) and the DAQ system of the FAST prototype (b). The dimensions of the optics are approximately $1.8 \mathrm{~m} \times 2.0 \mathrm{~m} \times 2.6 \mathrm{~m}(\mathrm{H} \times \mathrm{W} \times \mathrm{L})$. The FAST prototype camera, consisting of a single $200 \mathrm{~mm}$ PMT and a UV transparent filter, was installed at the focal plane of the telescope.

The EUSO-TA telescope is hosted in a small hut in front of the TA FD building at the Black Rock Mesa site. Its optics consist of two $1 \mathrm{~m}^{2}$ Fresnel lenses, with a UV transparent acrylic plate placed at the diaphragm for protection as shown in Figure $\mathbb{W}(\mathrm{a})$. For the purpose of the FAST test we installed a $200 \mathrm{~mm}$ PMT (R5912-03, Hamamatsu) at the focal plane of the telescope. A UV band-pass filter (Schott MUG-6 glass) was placed in front of the PMT to reduce the night-sky background. The PMT was equipped with an AC-coupled active base (E7694-01, Hamamatsu) to maintain stable gain under the high current expected during operation. To track the PMT response, we attached a YAP pulsed light source consisting of a $\mathrm{YAlO}_{3}$ :Ce scintillator crystal excited by a $50 \mathrm{~Bq}^{241} \mathrm{Am}$ source to the PMT's surface. The electronics and Data Acquisition System (DAQ) of the FAST prototype was built from commercial modules as summarized in Figure W(b). The FAST DAQ was remotely controlled via a wireless network.

\section{Measurements at the TA site}

The FAST prototype operated for 19 days in April and June 2014 during clear, moonless nights for a total of 83 hours. Several measurements were performed to validate the FAST concept, including studies of the night-sky background, of the stability of the YAP signal and of distant UV laser shots.

\subsection{Night-sky background and stability}

The average current of a pixel in any FD telescope is dominated by the night-sky background (NSB), typically $\sim 100$ photons $/ \mathrm{deg}^{2} / \mathrm{m}^{2} / \mu$ s. Current generation FD telescopes have a small FOV when compared to the $\sim 7^{\circ}$ radius of the FAST prototype. Since the average pixel current is proportional to the light-collecting area of the telescope and the pixel solid angle, we expect a significantly larger current in the FAST prototype. 


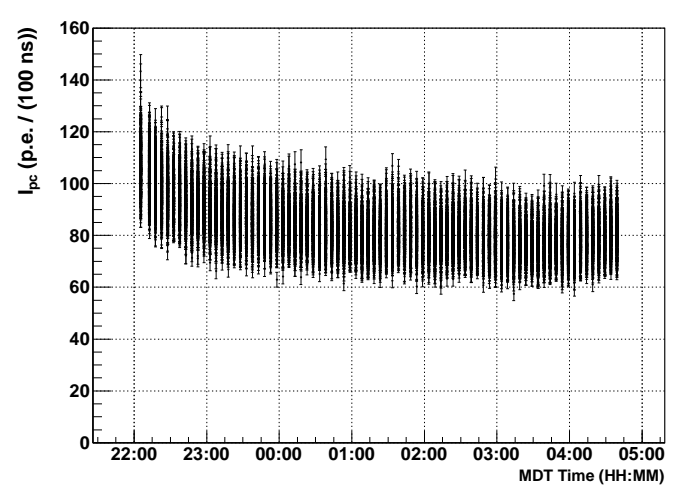

(a) Current monitor

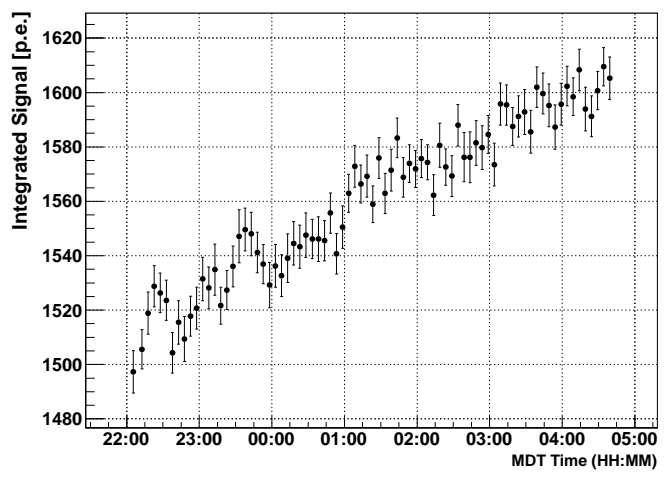

(b) Gain monitor

Figure 2: Stability of the current (a) and variation in the YAP signal intensity (b) during a seven hour data taking run.

AC coupling of the FAST PMT does not allow a direct measurement of the average current. However, fluctuations of the NSB are recorded as fluctuations of the PMT pedestal, whose variance is linearly related to the average current [Q]. With the shutter closed, the average signal, 15 photoelectrons (p.e.)/100 ns, is dominated by the noise in the FAST electronics chain. When exposed to the NSB, a current of 115 p.e./100 ns is measured, indicating that the electronic noise is negligible with respect to the NSB. The r.m.s. fluctuations of the NSB, $\sigma \sim 11$ p.e./100 ns, dictate the sensitivity of this FAST prototype. The evolution of the current during seven hours of continuous data taking is shown in Figure $\mathbb{Z}(\mathrm{a})$. A smooth decrease in the current as a function of time is observed, representing the change in the NSB during operation.

The FAST PMT gain was monitored during data taking with the stable light pulses provided by the YAP source. The measured variation in the YAP signal intensity during a night is shown in Figure $\square(b)$. The overall change is small $(\sim 7 \%)$ and consistent with the known temperature dependence of the PMT gain $\left(\sim-1 \%{ }^{\circ} \mathrm{C}^{-1}\right)$.

\subsection{Detection of distant laser shots}

UV laser shots are routinely used for calibration of FD telescopes and atmospheric monitoring [ए]] [U]]. While traveling up through the atmosphere, the laser light scatters on air molecules and aerosol particles into the FD field of view, producing signals similar to a UHECR shower. The TA site is equipped with a Central Laser Facility (CLF), located about $21 \mathrm{~km}$ from the Black Rock Mesa site. It consists of a $355 \mathrm{~nm}$ UV laser which fires 300 vertical shots every 30 minutes during data taking. In addition, a Portable UV Laser System (PLS) [ए2] can be deployed at different locations in the TA site. Both systems provide laser pulses of $\sim 2.2 \mathrm{~mJ}$ energy, approximately equivalent in intensity to a $\sim 10^{19.2} \mathrm{eV}$ shower. We made extensive use of these laser facilities to characterize the performance of the FAST prototype.

The signal measured by FAST for a single PLS shot is shown in Figure B](a), with the PLS located at a distance of $6 \mathrm{~km}$. The signal is well above the NSB level, and individual pulses were detected with $100 \%$ efficiency. We used this data to calibrate the relative timing between FAST and the TA FD by comparing the GPS time recorded by the two detectors for the same laser shot. 


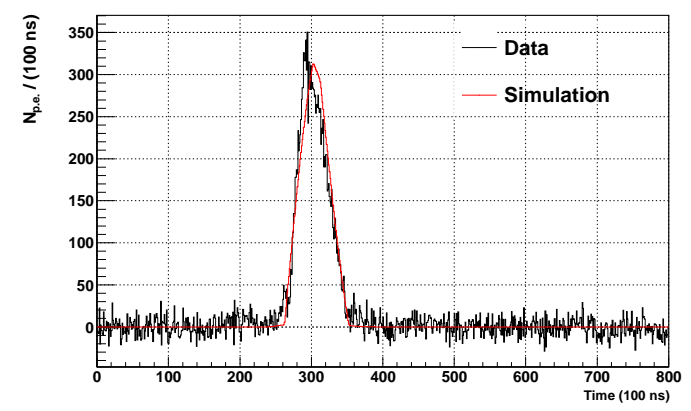

(a) PLS signal

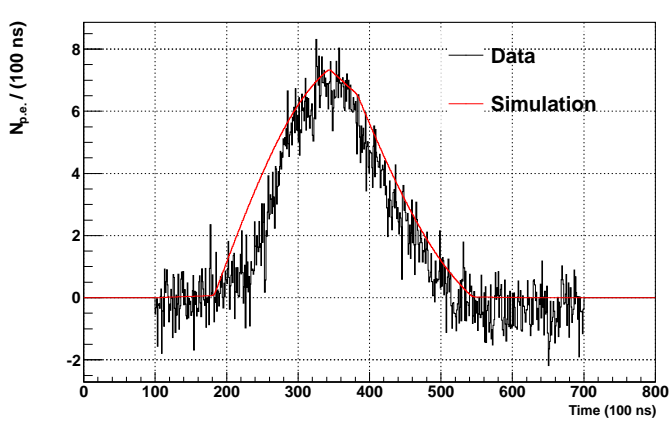

(b) CLF signal

Figure 3: FADC signals corresponding to PLS (a) and CLF (b) laser shots from a distance of $6 \mathrm{~km}$ and 21 $\mathrm{km}$, respectively. For the CLF, 233 laser shot signals are averaged to improve the sensitivity, since a single laser shot is at the limit of detection. The red curves show the expected signal from simulations of a $2.2 \mathrm{~mJ}$ vertical laser. The PLS simulated signal was normalized to fit the measured peak.

An offset was expected, since the external trigger to the FAST DAQ required some processing time in the TA trigger board. The distribution of the difference between the FAST and TA fluorescence detector GPS times is shown in Figure 田(a). An offset of $20.86 \mu$ s was measured, attributed to the TA trigger processing time. The r.m.s. of $\sim 100 \mathrm{~ns}$ is consistent with the GPS resolution, and adequate for the purpose of the FAST prototype test. A precise measurement of this relative timing was essential in the search for UHECR showers presented in Section $⿴$.

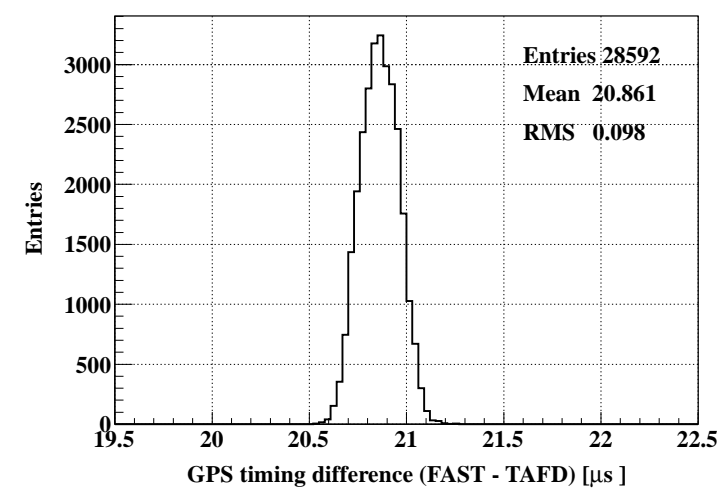

(a) GPS timing difference

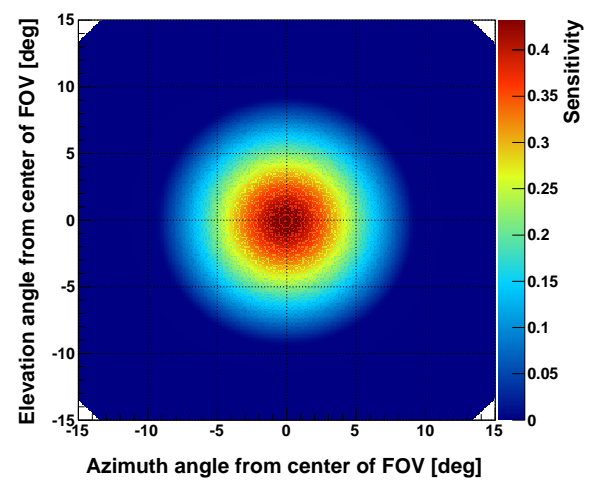

(b) Directional efficiency

Figure 4: Difference between the TA FD and the FAST prototype GPS time for laser shots (a). Efficiency of the optics of the FAST prototype as a function of the angle to the optical axis (b), obtained with a ray tracing simulation of the telescope.

We also performed measurements with the CLF, whose laser shots passed right through the center of the FAST FOV. The CLF signal was expected to be attenuated to the limit of detectability due to its distance from the FAST prototype and light absorption in the atmosphere. Individual CLF laser pulses could not be resolved. However, a clear signal was observed when averaging over many laser shots as shown in Figure B(b). The average signal amplitude was found to be 
7 p.e. $/ 100 \mathrm{~ns}$, indeed too small to allow for the detection of individual shots (compared with the NSB fluctuation, see Section B.]).

A simulation of the response of the FAST prototype to laser shots was performed to compare with the PLS and CLF data. For this purpose, the efficiency of the EUSO-TA telescope as a function of angle was obtained from a ray-tracing simulation of the Fresnel lenses as shown in Figure The FAST simulation included the wavelength dependent quantum efficiency of the FAST PMT (measured in a dedicated laboratory setup before installation) and realistic light attenuation in the

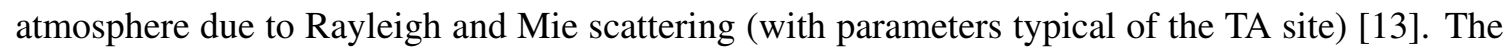
simulated signal for PLS and CLF laser shots is given in Figure [3. In both cases the amplitude and time width are in good agreement with the measurements.

\section{Detection of UHECR showers}

Detection of very energetic showers $\left(>10^{19} \mathrm{eV}\right)$ in the limited running time of the FAST prototype was unlikely. However, we expected to observe a few lower energy, close-by showers. A search was performed, driven by well reconstructed TA FD events which generated an external trigger for the FAST DAQ.

First we selected TA FD events with a reconstructed shower geometry passing through the FOV of the FAST prototype as shown in Figure 5(a). We then searched the corresponding FAST FADC traces for pulses with a maximum signal greater than $5 \sigma$, where $\sigma$ was calculated from the pedestal r.m.s. of the first $10 \mu \mathrm{s}$ of the trace as shown in Figure 5(b). The search was performed in a time interval of $70 \mu \mathrm{s}$, positioned in the trace according to the relative timing between FAST and the TA FD (Section [.2). We found 16 shower candidates in the 83 hour data-set, with an estimated background of $<1$ event.

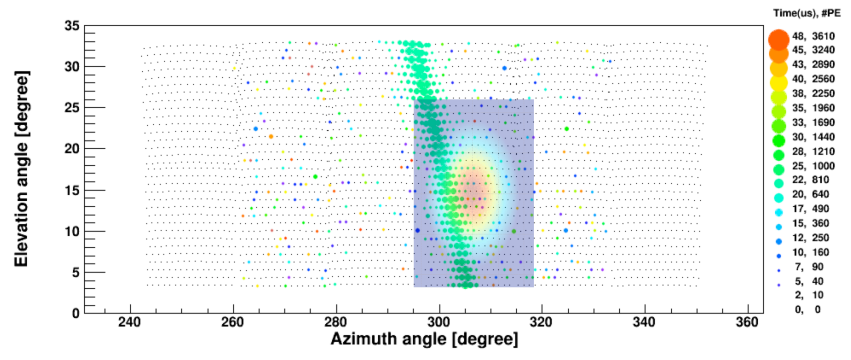

(a) TA FD

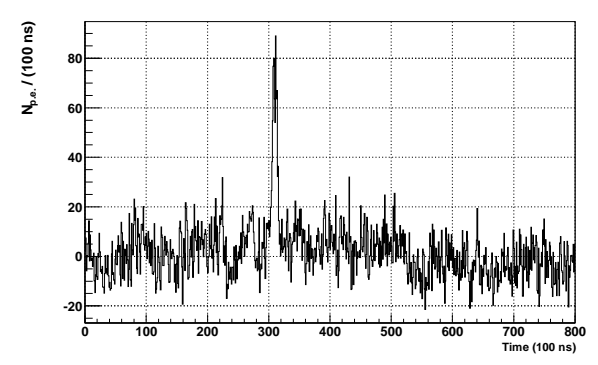

(b) FAST Prototype

Figure 5: A $10^{18} \mathrm{eV}$ shower simultaneously detected by the TA FD (a) and the FAST prototype (b). The TA FD display shows the number of observed photo-electrons (point size) and timing (point color) for each PMT. The track at the center of the display corresponds to the shower signal. The FAST FOV is indicated in (a).

Although small, this sample provides an estimate of the sensitivity of the FAST prototype. The correlation between the impact parameter (i.e. the distance of closest approach of the shower axis with respect to the FAST prototype) and the energy of the 16 showers is plotted in Figure 6 (a), with shower parameters given by the standard reconstruction of the TA FD [14]]. At any given energy, 


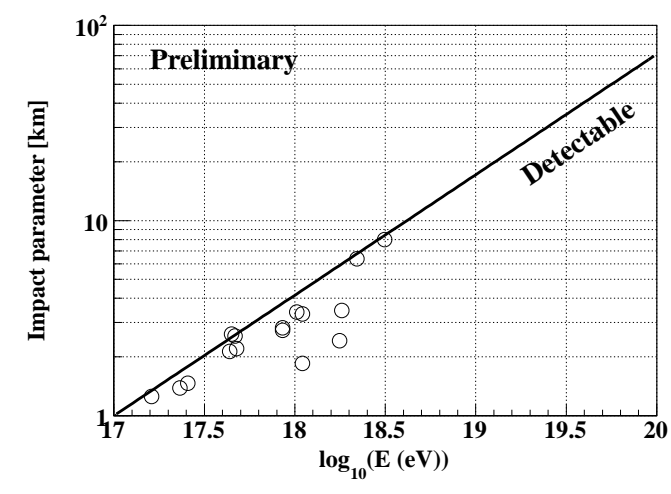

(a) Detectable distance

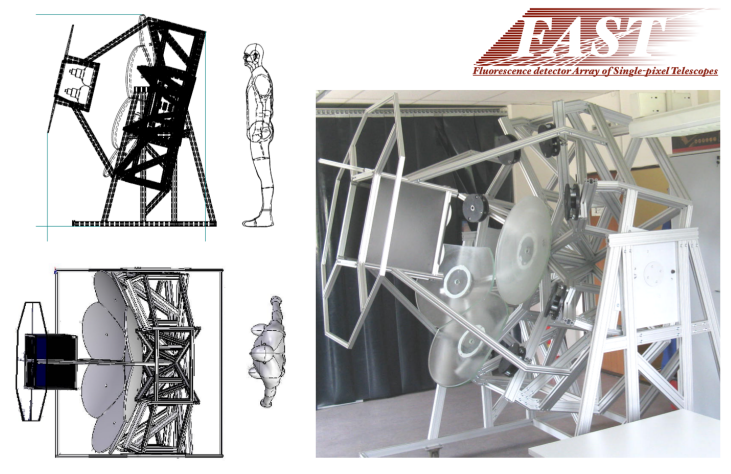

(b) Full-scale FAST prototype

Figure 6: Correlation between the impact parameter and energy of the 16 cosmic ray shower candidates detected by FAST (a). Both shower parameters were obtained from the TA standard reconstruction. The line indicates the maximum detectable distance consistent with our limited data set. A full-scale FAST prototype under development (b).

we expect showers to be detected up to a maximum impact parameter, $r_{\mathrm{det}}$. An approximate $r_{\mathrm{det}}$ bounding our limited data set is indicated by the line in Figure 6(a).

\section{Conclusions}

We have presented a novel concept for an air shower fluorescence detector, which features just a few pixels covering a large field of view. The FAST concept may be used in the next generation of UHECR experiments, which will require low cost detectors to achieve an order of magnitude increase in aperture. We reported first results of the FAST prototype at the Telescope Array site, where we installed a $200 \mathrm{~mm}$ PMT in the existing EUSO-TA telescope prototype optics. The FAST prototype took data during 19 nights, for a total of 83 hours. The detector operated under a variety of conditions typical of field deployment, demonstrating a remarkable stability and robustness. UV lasers placed at several kilometres distance were clearly detected by the FAST prototype, providing an estimate of its sensitivity. We also searched for UHECR showers detected by FAST in time coincidence with the TA FD, and found 16 highly significant candidates. These results give us confidence in the validity of the concept and its expected performance. Motivated by these encouraging results, a full-scale FAST prototype is under development; a design consisting of a $30^{\circ} \times 30^{\circ}$ FOV telescope of $1 \mathrm{~m}^{2}$ effective area, with a $2 \times 2$ PMT camera and a segmented spherical mirror of $1.6 \mathrm{~m}$ diameter is shown in Figure $6(\mathrm{~b})$.

\section{Acknowledgements}

This work was supported in part by NSF grant PHY-1412261 and by the Kavli Institute for Cosmological Physics at the University of Chicago through grant NSF PHY-1125897 and an endowment from the Kavli Foundation and its founder Fred Kavli. This project has been partially funded by the Italian Ministry of Foreign Affairs and International Cooperation. The Czech authors gratefully acknowledge the support of the Ministry of Education, Youth and Sports of the 
Czech Republic project No. LG13007. This work was supported by the Japan Society for the Promotion of Science through the Grant-in-Aid for Young Scientist (A) Grant Number 15H05443. TF was supported by the Japan Society for the Promotion of Science Fellowship for Research Abroad H25-339. The authors thank the Telescope Array and the JEM-EUSO Collaborations for providing logistic support and part of the instrumentation to perform the FAST prototype measurements. They also thank the Pierre Auger Collaboration for fruitful discussions.

\section{References}

[1] A. A. Watson, High-energy cosmic rays and the Greisen-Zatsepin-Kuz'min effect, Rep.Prog.Phys. 77 (2014) 036901,

[2] Pierre Auger Collaboration, A. Aab et al., The Pierre Auger Cosmic Ray Observatory, accepted for publication in Nucl.Instrum.Meth. A (2015) [1502.01323].

[3] H. Tokuno, Y. Tameda, M. Takeda, K. Kadota, D. Ikeda, et al., New air fluorescence detectors employed in the Telescope Array experiment, Nucl.Instrum.Meth. A676 (2012) 54-65, [1201.0002]].

[4] Telescope Array Collaboration, T. Abu-Zayyad et al., The surface detector array of the Telescope Array experiment, Nucl.Instrum.Meth. A689 (2012) 87-97,

[5] K.-H. Kampert and P. Tinyakov, Cosmic rays from the ankle to the cutoff, C.R.Phys. 15 (2014) 318-328, [11405.05/5]].

[6] JEM-EUSO Collaboration, Y. Takahashi, The JEM-EUSO mission, New J.Phys. 11 (2009) 065009, $[09010.418$ ].

[7] T. Fujii, M. Malacari, M. Bertaina, M. Casolino, B. Dawson, et al., Detection of ultra-high energy cosmic ray showers with a single-pixel fluorescence telescope, submitted to Astropart.Phys. (2015) [1504.00692].

[8] JEM-EUSO Collaboration, Y. Takizawa et al., The TA-EUSO and EUSO-Balloon optics designs, Proc. of the 33th International Cosmic Ray Conference, Rio de Janeiro, Brazil (2013) ID0832 [1307.707]].

[9] H. Gemmeke, M. Kleifges, and A. Menshikov, Statistical calibration and background measurements of the Auger fluorescence detector, Forschungszentrum Karlsruhe Report FZKA (2003) 891-894.

[10] B. Fick, M. Malek, J. A. J. Matthews, J. Matthews, R. Meyhandan, et al., The Central Laser Facility at the Pierre Auger Observatory, J.Instrum. 1 (2006), no. 11 P11003-P11003.

[11] S. Udo, R. Cady, M. Fukushima, J. N. Matthews, T. Jason, et al., The Central Laser Facility at the Telescope Array, Proc. of the 30th International Cosmic Ray Conference, Merida, Mexico 5 (2007) 1021-1024.

[12] Telescope Array Collaboration, K. Yamazaki et al., Calibration for the Telescope Array Fluorescence Detector using Portable UV Laser System, Proc. of the 33th International Cosmic Ray Conference, Rio de Janeiro, Brazil (2013) ID0526.

[13] T. Tomida, Y. Tsuyuguchi, T. Arai, T. Benno, M. Chikawa, et al., The atmospheric transparency measured with a LIDAR system at the Telescope Array experiment, Nucl.Instrum.Meth. $\mathbf{A 6 5 4}$ (2011) 653-660, [1]09.1196].

[14] Telescope Array Collaboration, T. Fujii et al., An event reconstruction method for the Telescope Array Fluorescence Detectors, AIP Conf.Proc. 1367 (2011) 149-152. 